\title{
Health related quality of life and comorbidity. A descriptive analysis comparing EQ-5D dimensions of patients in the German disease management program for type 2 diabetes and patients in routine care
}

Dominik Ose ${ }^{1 *}$, Antje Miksch¹, Elisabeth Urban ${ }^{1}$, Iris Natanzon ${ }^{1}$, Joachim Szecsenyi ${ }^{1}$, Cornelia Ursula Kunz ${ }^{2}$ and Tobias Freund ${ }^{1}$

Background: The co-occurance of multiple medical conditions has a negative impact on health related quality of life (HRQoL) for patients with type 2 diabetes. These patients demand for intensified care programs. Participation in a disease management program (DMP) for type 2 diabetes has shown to counterbalance this effect. However, it remains unclear which dimensions of HRQoL are influenced by the DMP. The aim of this study was to explore the HRQoL dimensions of patients with type 2 diabetes in the German DMP and patients in routine care (RC).

Methods: This analysis is part of a comparative evaluation of the German DMP for patients with type 2 diabetes. A questionnaire, including the HRQoL measure EQ-5D, was mailed to a random sample of 3,546 patients with type 2 diabetes (59.3\% female). The EQ-5D dimensions were analyzed by grouping patients according to their participation in the German DMP for diabetes into DMP and RC.

Results: Compared to patients in DMP, patients in $\mathrm{RC}$ reported more problems for the dimensions mobility $(\mathrm{P}<$ $0.05)$, self care $(P<0.05)$ and performing usual activities $(P<0.01)$. Depending on the number of other conditions, remarkable differences for reporting "no problems" exist for patients with six or more comorbid conditions regarding the dimensions mobility $(R C=8.7 \%$, $D M P=32.3 \%)$, self care $(R C=43.5 \%$, $D M P=64.5 \%)$, usual activities $(\mathrm{RC}=13.0 \%, \mathrm{DMP}=33.9 \%)$ and anxiety or depression $(\mathrm{RC}=37.0 \%, \mathrm{DMP}=48.4 \%)$.

Conclusion: Patients participating in the German DMP for type 2 diabetes mellitus show significantly higher ratings of their HRQoL in the dimensions mobility, self care and performing usual activities compared to patients in RC. This difference can also be observed in patients with significant comorbidities. As these dimensions are known to be essential for diabetes care, the German DMP may contribute to improved care even for comorbid diabetes patients.

\section{Background}

A special disease management program (DMP) for patients with type 2 diabetes has been in place in Germany since 2003. This primary care-based continuous program is accessible for all patients with type 2 diabetes insured within the statutory health insurance.

\footnotetext{
* Correspondence: dominik.ose@med.uni-heidelberg.de 'Department of General Practice and Health Services Research, University Hospital of Heidelberg, Vosstrasse 2, D-69115 Heidelberg, Germany Full list of author information is available at the end of the article
}

Currently more than 3,200,000 patients with type 2 diabetes participate in the DMP. Important elements of this approach like evidence-based clinical guidelines or transfer between different levels of care are defined by a national expert group. In contrast to vendor-supported programs in the United States, general practitioners in small- to medium-sized practices have an important role in coordinating the care of enrolled patients. Previous evaluations of this program show positive results regarding quality of care and health-related quality of life [1-3]. 
Nevertheless, dealing with co-morbidity is an enormous challenge in the German DMP for type 2 diabetes. Up to 90 percent of enrolled patients suffer from one or more co-occuring medical conditions [4]. Comorbidity is demanding for both healthcare systems and patients. It implies complex clinical management and increasing health care costs [5-7] as well as impaired health-related quality of life (HRQoL). It is known that the presence of co-occuring medical conditions has a negative impact on HRQoL for patients with type 2 diabetes [8-13]. We could previously show that the German DMP for type 2 diabetes may help to counterbalance the negative effect of comorbidity on HRQoL [14]. However, it remains unclear which dimensions of HRQoL are influenced by DMP. Therefore the aim of this analysis was to assess differences in the five dimensions of a valid multi-dimensional instrument for HRQoL (EQ-5D) between patients participating in the German DMP for type 2 diabetes and patients in routine care $(\mathrm{RC})$.

\section{Methods}

This analysis was performed as part of the ELSID study (Evaluation of a Large-scale Implementation of Disease management programs; 2005-2007). This observational study aims to compare the care provided within the DMP with routine care $(\mathrm{RC})$. The study protocol was approved by the ethics committee of the University of Heidelberg [15].

All of the participants in this study were insured by 1 large statutory regional healthcare fund called the Allgemeine Ortskrankenkasse (AOK), which covers about $40 \%$ of the German population. Patients were identified from routine claims data of this healthcare fund. To be included in the study, patients had to be older than age 50 years and be receiving a prescription for antidiabetic medication (oral antidiabetic drugs or insulin) in the first half-year of 2005. Patients in the DMP group had to be enrolled in the program by December 31, 2005, regardless of how long they had participated in the program prior to that date. Patients in the non-DMP group were not enrolled in the DMP before this appointed date.

Overall $n=20,625$ patients $(59.2 \%$ female $)$ were included in the ELSID-study. The population for the presented survey was a random sample of 3,546 patients (59.3\% female) from all study patients. In 2006, these patients received questionnaires with a cover letter sent by their health insurance provider. Details of the data acquisition have already been published [16].

In this survey we used the EQ-5D, a validated generic instrument for measuring HRQoL, which is available in more than 50 languages. The self-report questionnaire consists of a descriptive system, which defines health in terms of five dimensions: mobility, self-care, usual activities, pain or discomfort, and anxiety or depression. Each dimension is divided into three levels, indicating no problem (1st level), some or moderate problems (2nd level) and extreme problems (3rd level). The level of problem, reported on each of the EQ-5D dimensions, determines a unique health state $[17,18]$. Further investigations have demonstrated the usefulness of EQ-5D in identifying determinants of health states $[19,20]$. The questionnaire also included questions on sociodemographic characteristics (age, gender, educational level, marital status, and household income), self-reported health information (weight, height, and smoking status) and a list of chronic conditions in lay language (hypertension, osteoarthrosis, cancer, previous stroke, coronary heart disease, COPD, asthma, heart failure, and previous heart attack).

The EQ-5D dimensions were analyzed by grouping patients according to their participation in the German DMP for diabetes into DMP and RC. To analyze differences between groups we compared the proportion of reported problems (none, moderate and extreme) and calculated chi-square tests for each dimension. To describe differences depending on comorbidity we compared the proportion of reporting "no problems" for each dimension between patient groups with different numbers of other conditions $(0,1,2,3,4,5,6$ and more). All analyses were conducted using SPSS (version 15.0).

\section{Results}

A total of 1,532 questionnaires were returned (response rate: $42.2 \%$ ). Valid data were available for 1,399 patients, more precisely 865 patients in DMP $(61,83 \%)$ and 534 $(38,17 \%)$ patients in RC. $53.6 \%$ of these were female and the mean age for the entire sample was $70.3( \pm 8.5)$ years. Significant differences between the two groups (DMP and RC) did not exist for the total sample but for some subgroups (Table 1).

The EQ-5D was completed by 1,291 patients. The analysis of EQ-5D dimensions showed significant differences for reporting problems in the dimensions mobility $(\mathrm{P}<0.05)$, self care $(\mathrm{P}<0.05)$ and performing usual activities $(\mathrm{P}<0.01)$. For the dimensions pain or discomfort and anxiety or depression we found no significant difference (Table 2). Depending on the number of cooccuring conditions differences for reporting "no problems" could be observed particularly for patients with six or more co-occuring conditions in the dimensions mobility $(\mathrm{RC}=8.7 \%, \mathrm{DMP}=32.3 \%)$, self care $(\mathrm{RC}=$ $43.5 \%, \mathrm{DMP}=64.5 \%)$, usual activities $(\mathrm{RC}=13.0 \%$, $\mathrm{DMP}=33.9 \%)$ and anxiety or depression $(\mathrm{RC}=37.0 \%$, DMP $=48.4 \%)($ Table 3$)$. However, these observed differences were not statistically significant. 
Table 1 Patient characteristics

\begin{tabular}{|c|c|c|c|c|c|c|c|c|c|c|c|c|c|c|c|c|}
\hline & & & \multicolumn{14}{|c|}{ Number of other chronic conditions } \\
\hline & \multicolumn{2}{|c|}{ Sample } & \multicolumn{2}{|c|}{ no } & \multicolumn{2}{|c|}{1} & \multicolumn{2}{|c|}{2} & \multicolumn{2}{|c|}{3} & \multicolumn{2}{|c|}{4} & \multicolumn{2}{|c|}{5} & \multicolumn{2}{|c|}{$6 \leq$} \\
\hline & DMP & $\mathrm{RC}$ & DMP & $\mathrm{RC}$ & DMP & $\mathrm{RC}$ & DMP & $\mathrm{RC}$ & DMP & $\mathrm{RC}$ & DMP & $\mathrm{RC}$ & DMP & $\mathrm{RC}$ & DMP & $\mathrm{RC}$ \\
\hline $\mathrm{N}$ & 865 & 534 & 66 & 40 & 189 & 111 & 214 & 140 & 178 & 91 & 93 & 64 & 60 & 42 & 65 & 46 \\
\hline Age (mean) & 70.2 & 70.5 & 72.4 & $68.6^{*}$ & 69.2 & 69.6 & 69.8 & 71.3 & 70,4 & 70,9 & 69.5 & 70.8 & 71.8 & 70.7 & 72.2 & 71.8 \\
\hline Age (SD) & \pm 8.3 & \pm 8.9 & \pm 8.0 & \pm 7.7 & \pm 8.0 & \pm 9.3 & \pm 8.5 & \pm 8.5 & $\pm 8,2$ & $\pm 8,9$ & \pm 7.6 & \pm 7.8 & \pm 9.0 & \pm 10.4 & \pm 8.9 & \pm 8.7 \\
\hline Female subjects (\%) & 53.8 & 53.4 & 53.0 & 55.0 & 55.6 & 47.7 & 57.9 & 58.9 & 51,7 & 59,3 & 47.3 & 50.0 & 56.7 & 40.5 & 44.6 & 56.5 \\
\hline Education $\leq 9$ years (\%) & 70.8 & 72.3 & 76.7 & 81.1 & 75.9 & 75.5 & 75.0 & 79.6 & 74,7 & $85.7^{*}$ & 76.1 & 76.7 & 76.8 & 86.1 & 79.7 & 79.1 \\
\hline Living in partnership (\%) & 65.7 & 62.9 & 55.4 & 52.6 & 64.9 & 64.2 & 61.9 & 62.0 & 66,7 & $52.8^{*}$ & 63.7 & 63.5 & 52.6 & 61.9 & 64.1 & 51.1 \\
\hline BMI (mean) & 30.3 & 30.3 & 29.1 & 30.3 & 29.4 & 30.2 & 30.5 & 29.3 & 30,7 & 30,4 & 31.3 & 30.1 & 31.0 & 33.2 & 30.2 & 31.4 \\
\hline $\mathrm{BMI}(\mathrm{SD})$ & \pm 5.8 & \pm 6.5 & \pm 5.2 & \pm 5.2 & \pm 5.0 & \pm 6.1 & \pm 5.7 & \pm 6.1 & $\pm 6,6$ & $\pm 7,5$ & \pm 5.7 & \pm 5.7 & \pm 6.6 & \pm 7.4 & \pm 6.0 & \pm 7.2 \\
\hline Hypertension (\%) & 71.3 & 72.1 & -——- & -——- & 24.3 & 20.7 & 62.1 & 63.6 & 74,2 & 73,6 & 77.4 & 82.8 & 91.7 & 69.0 & 87.7 & 91.3 \\
\hline Osteoarthrosis (\%) & 57.2 & 56.7 & - - & -- & 57.7 & 49.5 & 79.0 & 77.9 & 83,7 & 89,0 & 87.1 & 90.6 & 85.0 & $90.5^{*}$ & 89.2 & 95.7 \\
\hline Coronary heart (\%) & 20.9 & 20.4 & -——— & -——- & 0.5 & $7.2^{*}$ & 7.0 & 7.1 & 24,2 & 17,6 & 50.5 & 42.2 & 51.7 & 40.5 & 67.7 & 67.4 \\
\hline Heart failure (\%) & 16.9 & 18.0 & -—— & -——- & 3.7 & 2.7 & 9.3 & 5.7 & 17,4 & 13,2 & 32.3 & 32.8 & 48.3 & 50.0 & 44.6 & $67.4^{*}$ \\
\hline COPD (\%) & 9.9 & 10.9 & -—— & -——- & 0.5 & $3.6^{*}$ & 4.2 & 3.6 & 10,7 & 9,9 & 25.8 & 25.0 & 13.3 & 16.7 & 38.5 & 37.0 \\
\hline Cancer (\%) & 7.6 & 9.0 & -——- & -——- & 3.2 & 1.8 & 5.6 & 4.3 & 6,7 & 11,0 & 16.1 & 17.2 & 13.3 & 4.8 & 20.0 & 37.0 \\
\hline Previous heart attack (\%) & 7.1 & 8.2 & -—— & -——- & 0.5 & 0.0 & 0.9 & $6.4^{*}$ & 8,4 & 8,8 & 18.3 & 10.9 & 11.7 & 19.0 & 29.2 & 26.1 \\
\hline Previous stroke (\%) & 6.0 & 6.6 & -——- & -——- & 1.1 & 2.7 & 3.7 & 2.1 & 9,0 & 7,7 & 6.5 & 12.5 & 15.0 & 11.9 & 16.9 & 19.6 \\
\hline Asthma (\%) & 4.2 & 4.5 & -—- & -—-— & 0.0 & 0.9 & 1.4 & 1.4 & 7,3 & 2,2 & 4.3 & 4.7 & 11.7 & 26.2 & 13.8 & 10.9 \\
\hline
\end{tabular}

\section{Discussion}

The analysis revealed differences between DMP and RC patients for the HRQoL dimensions "mobility", "self care", and "performing usual activities". DMP patients reported significantly less problems in these dimensions.
These differences could also be observed in patients with significant comorbidities.

These results are in line with our previous findings [14] and provide additional understanding of how the German DMP for patients with type 2 diabetes mellitus

Table 2 Analysis of EQ-5D dimensions $(n=1291)$

\begin{tabular}{|c|c|c|c|c|c|c|}
\hline \multirow[t]{2}{*}{ Dimension } & \multicolumn{2}{|c|}{ Sample } & \multicolumn{3}{|c|}{ Problems } & \multirow[t]{2}{*}{ p-value* } \\
\hline & $\mathrm{RC}$ & DMP & level & $\mathrm{RC}$ & DMP & \\
\hline & $n$ & $n$ & & $\%$ & $\%$ & \\
\hline \multirow[t]{3}{*}{ mobility } & 482 & 809 & no & 51,3 & 54,6 & 0.041 \\
\hline & & & some & 47,6 & 45,2 & \\
\hline & & & extreme & 1,0 & 0,1 & \\
\hline \multirow[t]{3}{*}{ self care } & 482 & 809 & no & 80,1 & 84,7 & 0.010 \\
\hline & & & some & 15,7 & 13,7 & \\
\hline & & & extreme & 4,1 & 1,6 & \\
\hline \multirow[t]{3}{*}{ performing usual activities } & 482 & 809 & no & 56,3 & 59,5 & 0.006 \\
\hline & & & some & 35,0 & 36,2 & \\
\hline & & & extreme & 8,7 & 4,3 & \\
\hline \multirow[t]{3}{*}{ pain/discomfort } & 482 & 809 & no & 19,0 & 19,4 & 0.088 \\
\hline & & & some & 65,6 & 69,5 & \\
\hline & & & extreme & 15,3 & 11,1 & \\
\hline \multirow[t]{3}{*}{ anxiety/depressed } & 482 & 809 & no & 67,5 & 66,9 & 0.682 \\
\hline & & & some & 28,4 & 29,8 & \\
\hline & & & extreme & 4,1 & 3,3 & \\
\hline
\end{tabular}


Table 3 No problems reported by number of other chronic conditions $(n=1291)$

\begin{tabular}{|c|c|c|c|c|c|c|c|c|c|c|c|c|}
\hline \multirow[t]{2}{*}{ number of other conditions } & \multicolumn{2}{|c|}{ sample } & \multicolumn{2}{|c|}{ mobility } & \multicolumn{2}{|c|}{ self care } & \multicolumn{2}{|c|}{ usual activities } & \multicolumn{2}{|c|}{ pain/discomfort } & \multicolumn{2}{|c|}{ anxiety/depression } \\
\hline & $\mathrm{RC}$ & DMP & $\mathrm{RC}$ & DMP & $\mathrm{RC}$ & DMP & $\mathrm{RC}$ & DMP & $\mathrm{RC}$ & DMP & $\mathrm{RC}$ & DMP \\
\hline & $n$ & $n$ & $\%$ & $\%$ & $\%$ & $\%$ & $\%$ & $\%$ & $\%$ & $\%$ & $\%$ & $\%$ \\
\hline 0 & 32 & 58 & 87,5 & 75,9 & 90,6 & 86,2 & 71,9 & 86,2 & 40,6 & 55,2 & 87,5 & 82,8 \\
\hline 1 & 99 & 179 & 75,8 & 74,9 & 91,9 & 93,9 & 77,8 & 82,1 & 37,4 & 36,9 & 80,8 & 76,0 \\
\hline 2 & 129 & 203 & 56,2 & 51,7 & 83,1 & 85,2 & 60,8 & 59,1 & 20,8 & 14,8 & 70,8 & 66,5 \\
\hline 3 & 82 & 163 & 50,0 & 53,4 & 79,3 & 85,9 & 58,5 & 52,8 & 11,0 & 9,8 & 63,4 & 62,0 \\
\hline 4 & 58 & 86 & 29,3 & 38,4 & 81,0 & 81,4 & 46,6 & 47,7 & 5,2 & 9,3 & 65,5 & 65,1 \\
\hline 5 & 36 & 58 & 27,8 & 32,8 & 75,0 & 75,9 & 33,3 & 27,6 & 5,6 & 3,4 & 52,8 & 60,3 \\
\hline$\geq 6$ & 46 & 62 & 8,7 & 32,3 & 43,5 & 64,5 & 13,0 & 33,9 & 2,2 & 4,8 & 37,0 & 48,4 \\
\hline
\end{tabular}

may improve HRQoL. However, methodological limitations of descriptive studies do not allow any conclusion on causal relationship. Nevertheless, the results give rise to the question as to which elements of the German DMP for type 2 diabetes could be contributing to the differences between groups. Indeed, we know that aspects like physical activities or social support can improve HRQoL [21-23]. Even so, it is uncertain which specific elements of DMP, such as structured care, regular follow ups or patient education programs, are responsible for the fact that patients - especially those with numerous other conditions - reported less problems.

Physical activity is an essential component of diabetes management [24]. Therefore, reporting less problems in the HRQoL dimension "mobility" may indicate that a higher proportion of DMP patients would be able to be physically active than RC patients. However, the actual level of physical activity of both groups was not assessed in this survey. "Self-care" as well as the ability to perform "usual activities" is seen to be crucial for independent living and HRQoL. The observed differences may therefore explain our previous results [14].

Our study is further limited by a moderate response rate. This rate might have been higher if the questionnaires had been sent out by the university department directly instead of the health fund. Due to a strict protection of data privacy we were not able to contact the patients directly. Also we do not know whether and how motivation to participate in a DMP affects HRQoL. Potential differences (age, gender, DMP status) between responders and non-responders may also affect our results. It should be considered that the age of patients in our sample is substantially higher than usually seen in diabetes studies.

\section{Conclusions}

Patients participating in the German DMP for type 2 diabetes mellitus show significantly higher ratings of their HRQoL in the dimensions mobility, self care and performing usual activities compared to patients in RC. This difference can also be observed in patients with significant comorbidities. As these dimensions are known to be essential for diabetes care, the German DMP may contribute to improved care even for comorbid diabetes patients.

\section{Acknowledgements}

This study is an investigator-initiated trial funded by the Federal Association of Statutory Regional Health Funds (AOK Bundesverband). Under the contract between the sponsor and the University of Heidelberg, full responsibility for the scientific work, the management of data and analysis and publication rests with the investigators. We are grateful to the regional health funds AOK Sachsen-Anhalt and AOK Rheinland Pfalz for support in sending out the study material to their policyholders and for the preparation of claims data for sampling purposes.

\section{Author details}

${ }^{1}$ Department of General Practice and Health Services Research, University Hospital of Heidelberg, Vosstrasse 2, D-69115 Heidelberg, Germany. ${ }^{2}$ Institute of Medical Biometry and Informatics, University Hospital of Heidelberg, Im Neuenheimer Feld 305, D-69120 Heidelberg, Germany.

\section{Authors' contributions}

AM and JS initiated, designed and coordinated the study. DO and TF carried out data-analysis and wrote the manuscript. CUK gave statistical advice. All authors, particularly IN and EU, read earlier versions of the manuscript, provided critical comments, and approved the final manuscript.

\section{Competing interests}

The authors declare that they have no competing interests.

Received: 1 February 2011 Accepted: 2 August 2011

Published: 2 August 2011

\section{References}

1. Schäfer I, Küver C, Gedrose B, Hoffmann F, Ruß-Thiel B, Brose HP, van den Busche $\mathrm{H}$, Kaduszkiewicz $\mathrm{H}$ : The disease management program for type 2 diabetes in Germany enhances process quality of diabetes care - a follow-up survey of patient's experiences. BMC Health Service Research 2010, 10:55.

2. Szecsenyi J, Rosemann T, Joos S, Peters-Klimm F, Miksch A: German diabetes disease management programs are appropriate for restructuring care according to the chronic care model: an evaluation with the patient assessment of chronic illness care instrument. Diabetes Care 2008, 31:1150-1154.

3. Stock S, Drabik A, Büscher G, Graf C, Ullrich W, Gerber A, Lauterbach KW, Lüngen M: German Diabetes Management Programs Improve Quality of Care And Curb Costs. Health Aff 2010 29:2197-2205. 
4. Altenhofen L, Blaschy S, Hagen B, Haß W, Kretschmann J: Qualitätsbericht 2006. [Disease Management Programme in Nordrhein]. Nordrheinische Gemeinsame Einrichtung; 2008.

5. Valderas JM, Starfield B, Sibbald B, Salisbury C, Roland M: Defining Comorbidity: Implications for Understanding Health and Health Services. Ann Fam Med 2009, 7:357-363.

6. Piette JD, Kerr EA: The Impact of Comorbid Chronic Conditions on Diabetes Care. Diabetes Care 2006, 29:725-731.

7. Halanych JH, Safford MM, Keys WC, Person SD, Shikany JM, Kim Yl, Centor RM, Allison Jj: Burden of comorbid medical conditions and quality of diabetes care. Diabetes Care 2007, 30:2999-3004.

8. Wexler DJ, Grant RW, Wittenberg E, Bosch JL, Cagliero E, Delahanty L, Blais MA, Meigs JB: Correlates of health related quality of life in type 2 diabetes. Diabetologia 2006, 49:1489-1497.

9. UK. Prospective Diabetes Study Group: Quality of Life in Type 2 Diabetic Patients Is Affected by Complications But Not by Intensive Policies to Improve Blood Glucose or Blood Pressure Control (UKPDS 37). Diabetes Care 1999, 22:1125-1136.

10. Maddigan SL, Feeny DH, Johnson JA: Health related quality of life deficits associated with diabetes and comorbidities in a Canadian National Population Health Survey. Qual Life Res 2005, 14:1311-1320.

11. Coffey JT, Brandle M, Zhou H, Marriott D, Burke R, Tabaei BP, Engelgau MM, Kaplan RM, Herman WH: Valuing Health-Related Quality of Life in Diabetes. Diabetes Care 2002, 25:2238-2243.

12. Wee HL, Chueng YB, Li SC, Fong KY, Thumboo J: The impact of diabetes mellitus and other chronic medical conditions on health-related Quality of Life: Is the whole greater than the sum of its parts? Health Qual Life Outcomes 2005, 3:2.

13. Koopmanschap M: Coping with Type II diabetes: the patient's perspective. Diabetologie 2002, 45:S18-S22.

14. Ose D, Wensing M, Szecsenyi J, Joos S, Hermann K, Miksch A: Impact of Primary Care-Based Disease Management on the Health-Related Quality of Life in Patients With Type 2 Diabetes Comorbidity. Diabetes Care 2009, 32:1594-1596.

15. Joos S, Rosemann T, Heiderhoff M, Wensing M, Ludt S, Gensichen J, Kaufmann-Kolle P, Szecsenyi J: ELSID-Diabetes study-evaluation of a large scale implementation of disease management programmes for patients with type 2 diabetes. In BMC Public Health. Volume 5. Rationale, design and conduct - a study protocol [ISRCTN08471887]; 2005:99.

16. Miksch A, Herman K, Rölz A, Joos S, Szecsenyi J, Ose D, Rosemann T: Additional impact of concomitant hypertension and osteoarthritis on quality of life among patients with type 2 diabetes in primary care in Germany - a cross-sectional survey. Health Qual Life Outcomes 2009, 7:19.

17. EuroQol Group: EuroQol - e new facility for the measurement of healthrelated quality of life. Health Policy 1990, 16:199-208.

18. Greiner W, Weijnen T, Nieuwenhuizen M, Oppe S, Badia X, Busschbach J, Buxton M, Dolan P, Kind P, Krabbe P, Ohinmaa A, Parkin D, Roset M, Sintonen H, Tsuchiya A, de Charro F: A single European currency for EQ5D health states. Results from a six-country study. Eur J Health Econom 2003, 4:222-231.

19. Barton GR, Sach TH, Doherty M, Avery AJ, Jenkinson C, Muir KR: An assessment of the discriminative ability of the EQ-5D index, SF-6D, and EQ VAS, using sociodemographic factors and clinical conditions. Eur J Health Econ 2008, 9:237-249.

20. Grandy S, Fox KM: EQ-5D visual analog scale and utility index values in individuals with diabetes and risk for diabetes. Findings from the Study to Help Improve Early evaluation and management of risk factors Leading to Diabetes (SHIELD). Health Qual Life Outcomes 2008, 6:18-25.

21. Chyun DA, Melkus GD, Katten DM, Price WJ, Davey JA, Grey N, Heller G, Wackers FJ: The association of psychological factors, physical activities, neuropathy, and quality of live in type 2 diabetes. Biol Res Nurs 2008, 7:279-288.

22. Acree LS, Longfors J, Fjeldstad AS, Fjeldstad C, Schank B, Nickel KJ, Montgomery PS, Gardner AW: Physical activity is related to quality in older adults. Health Qual Life Outcomes 2006, 4:37.

23. Göz F, Kraoz S, Goz M, Ekiz S, Cetin I: Effects of the diabetic perceived support on their quality-of-life. J Clin Nurs 2007, 16:1353-1360.

24. Sigal JF, Kenny GP, Wasserman DH, Castaneda-Sceppa C: Physical Activity/ Exercise and Type 2 Diabetes. Diabetes Care 2004, 10:2518-2539.

\section{Pre-publication history}

The pre-publication history for this paper can be accessed here: http://www.biomedcentral.com/1472-6963/11/179/prepub

doi:10.1186/1472-6963-11-179

Cite this article as: Ose et al:: Health related quality of life and comorbidity. A descriptive analysis comparing EQ-5D dimensions of patients in the German disease management program for type 2 diabetes and patients in routine care. BMC Health Services Research 2011 11:179.

\section{Submit your next manuscript to BioMed Central and take full advantage of:}

- Convenient online submission

- Thorough peer review

- No space constraints or color figure charges

- Immediate publication on acceptance

- Inclusion in PubMed, CAS, Scopus and Google Scholar

- Research which is freely available for redistribution

Submit your manuscript at www.biomedcentral.com/submit
Ciomed Central 